Revista Educación 25(2): 67-85, 2001

\title{
UNA CONSTRUCCIÓN METODOLÓGICA PARA COMPARTIR DESDE LA INVESTIGACIÓN CUALITATIVA
}

\author{
Marta Eugenia Sánchez González
}

Resumen: En este artículo sintetizo el proceso metodológico que fui construyendo durante la investigación que desarrollé en un aula de Primer año de I Ciclo durante el año 1999 y que titulé "Una nueva mirada a los procesos de lectura y escritura". El principal objetivo de ese estudio fue el develar los aspectos que promueven o dificultan la innovación de los procesos de lectura y escritura en ese nivel de la educación costarricense y con base en esa información diseñar propuestas de formación y actualización a los docentes y a las docentes.

Esa investigación de carácter cualitativo, etnográfico fue asumiendo elementos del estudio de casos, de la hermeneútica y de la fenomenología.

La construcción de la metodología fue fascinante, un proceso sumamente creativo. El volver a la escuela donde cursé mi educación primaria, esta vez, con una nueva mirada, me dio la oportunidad de aprender nuevamente de esa institución educativa. Ese espacio de aprendizaje me brindó una oportunidad para describir, analizar, interpretar y construir los significados desde las prácticas pedagógicas que abi tenían lugar.

Fue con base en la observación participante, en la entrevista en profundidad y en la entrevista coloquial, en el análisis de documentos, en la fotografía y en el vídeo que elaboré las notas crudas, luego las notas cocidas. Con base en esos registros diseñé varias matrices que me permitieron poco a poco, en un proceso de reflexión y valoración de la información, organizar los datos a la luz de la preguntas que orientaban la investigación. La organización de la información me orientó en la elaboración de los cinco apartados que dan unidad a la información recabada durante el proceso de investigación y que permiten hallazgos conducentes al logro del objetivo planteado.

\section{Introducción}

Pienso que pocas veces, como educadores o investigadores, nos detenemos a analizar el potencial que nos ofrece la escuela para aprender nuevamente. Si recordamos nuestra infancia, como niños y niñas disfrutamos de los aprendizajes que de manera integral nos ofreció esa institución educativa. Fue en ese espacio donde aprendimos a convivir con nuestros maestros y maestras, con nuestros amigos, amigas, compañeros y compañeras. En el "nicho del aula" tal y como llaman Goetz y LeCompte (1988) a ese espacio de aprendizaje, fue donde adquirimos nuestros primeros conocimientos. Más tarde, como profesionales podemos volver a ella, con nuevas experiencias, a aprender nuevamente, esta vez con una nueva mirada, de las prácticas pedagógicas que ahí se generan.

Esa nueva oportunidad de aprender de la escuela nos permite describir, analizar, interpretar y construir significados desde las prácticas pedagógicas que en ella hoy día tienen lugar y de esa manera, ofrecer elementos para que el encuentro de la teoría con la labor del maestro y la maestra en el aula, lleven a la innovación de los procesos educativos y por consiguiente, resulten más significativos para los actores y actoras de los procesos que en ese espacio tienen lugar.

Debido a lo anterior, fue que en 1998 , al incorporarme al Programa de Doctorado 
en Educación que ofrece la Universidad Estatal a Distancia, tuve de nuevo la oportunidad de volver al aula, regresar a la escuela donde cursé mi educación primaria, esta vez, como una profesional que deseaba, mediante la investigación, aprender nuevamente de esa institución educativa. Fue así como desarrollé una investigación cualitativa, etnográfica donde incorporé elementos del método fenomenológico y del método hermeneútico. El estudio lo llevé a cabo en un grupo de Primer año de I Ciclo movida por el deseo de develar aquellos aspectos que promueven o dificultan la innovación de los procesos de lectura y escritura para posteriormente, con base en esos descubrimientos, diseñar propuestas de formación y actualización de docentes que respondan a las necesidades y demandas que nuestros maestros y escuelas plantean, para de esa manera, favorecer la innovación de los procesos de lectura y escritura en nuestras instituciones de I y II Ciclos. Ese problema de investigación, surgió debido a que desde 1990 con la Política Curricular hasta hoy con la Política Educativa hacia el Siglo XXI, son innumerables los esfuerzos realizados tanto por el Ministerio de Educación Pública como por las universidades, por formar y actualizar a los docentes y las docentes de I y II Ciclos en el desarrollo de planes y programas de estudio orientados hacia la construcción del conocimiento sin lograr los cambios esperados en la práctica pedagógica que se lleva a cabo en nuestras aulas.

En el desarrollo de la investigación fui trazando la ruta que me permitiera recabar la información que requería para mi estudio, analizarla, interpretarla y con base en esa interpretación, construir los significados que emergieron en ese proceso. Lo anterior debido a que el diseño de este tipo de investigación no se elabora de una vez por todas, sino que se va construyendo poco a poco como producto de la interacción del investigador con su objeto de estudio. Esa construcción fue fascinante, sumamente creativa por lo que deseo compartir la metodología construida en el transcurso de ese estudio. Lo anterior, con el propósito de que la ex- periencia que comparto sirva de motivación e invite a educadores e investigadores a volver a la escuela, regresar a ella y descubrir todo el potencial de aprender que esa institución pone a nuestro alcance.

\section{Hacia la construcción del camino por seguir}

\section{Tipo de investigación}

La investigación llevada a cabo "Una nueva mirada de los procesos de lectura y escritura", se enmarca dentro de la corriente de la investigación cualitativa. Este tipo de investigación surge desde principios de siglo pero no es sino hasta las dos últimas décadas, con la formalización de los métodos cualitativos, que ha adquirido mayor relevancia en el estudio de los fenómenos del aula (Campos, 1998).

La incorporación de la metodología cualitativa al estudio de los fenómenos educativos se debe a múltiples factores, entre ellos se puede destacar un replanteamiento de carácter epistemológico sobre algunos presupuestos que guiaban anteriores investigaciones y su evolución se ve propiciada por su práctica en disciplinas afines al campo de la educación como la sociología, la psicología y la antropología entre otras (Colás y Buendía, 1994).

Shagoury y Miller (1993) consideran que los profesores y las profesoras cambian las formas de trabajo con sus alumnos y alumnas al mirar el aula de otra manera, de una manera sistemática que hace uso de la investigación. Para las autoras mencionadas la investigación que realizan los profesores y las profesoras está muy bien fundamentada, su base es sólida pues conocen las escuelas donde laboran, a sus colegas, los planes que orientan el trabajo no sólo de la escuela sino también del aula, la comunidad educativa y el entorno social en que se encuentra la institución educativa de la que forma parte.

De acuerdo con esas autoras la investigación que desarrollamos los docentes y las 
docentes ha llevado a un constante descubrimiento. Es un proceso maravilloso, fascinante, que requiere ser repensado constantemente en tanto nos permite no sólo comprender mejor la disciplina que enseñamos sino también revisar las preguntas, los cuestionamientos que el trabajo cotidiano plantea, problematizar lo que consideramos obvio en la actividad pedagógica, así como los procedimientos utilizados al analizar el trabajo realizado por los alumnos y las alumnas. Debido a lo anterior, la investigación así entendida nunca termina, siempre es posible descubrir nuevas rutas, otros caminos para saber qué es lo que está sucediendo en las aulas, surgen nuevas interrogantes que se desea contestar con el propósito de lograr el cambio, la innovación. Y por consiguiente lograr que los procesos de enseñanza y de aprendizaje sean significativos para los actores y las actoras de los mismos.

Guba y Lincoln (1982-1983) citados por Colás y Buendía (1994) señalan entre las características de la metodología cualitativa, las siguientes:

1. Concepción múltiple de la realidad: se asume que existen múltiples realidades que sólo se pueden estudiar holísticamente, es decir como un todo.

2. El principal objetivo científico será la comprensión de los fenómenos. Esto se logrará mediante el análisis de las percepciones e interpretaciones de los sujetos que intervienen en la acción educativa.

3. Investigador y objeto de investigación están interrelacionados, en continua interacción e influencia mutua.

4. El objetivo de la investigación es desarrollar un cuerpo de conocimientos ideográficos que describan los casos individuales.

5. La simultaneidad de los fenómenos e interacciones mutuas en el hecho educativo hacen imposible distinguir las causas de los efectos.

6. Procura alternativas para la formación y actualización de docentes de la educación general básica.
Por su lado, la investigación etnográfica de acuerdo con Campos (1998) se caracteriza por:

- Captar el fenómeno educativo en el ambiente natural como es el aula, en una institución determinada.

- Tomar en cuenta el punto de vista del observado.

- Captar la historia del fenómeno observado.

- Registrar los eventos objeto de la observación.

- Utilizar materiales para la recolección de la información como mapas, fotografías, videos y grabaciones, entre otros.

La concepción de investigación etnográfica que se asume al trabajar en el aula, es una concepción epistemológica que implica "aceptar conscientemente una correspondencia entre teoría, método y procedimientos" (Edwards, 1991:19), lo que de acuerdo con la misma autora, significa explicitar cómo se define la relación entre el sujeto y el objeto de conocimiento.

Lo anterior lleva a considerar la investigación como una construcción en la cual los elementos constituyentes no son rígidos ni planeados de una vez por todas sino, por el contrario, el diseño que orienta esta investigación reconoce la importancia de las interconexiones e interacciones que se dan entre los diferentes componentes del mismo (Maxwell, 1996). La estructura de este modelo de investigación es flexible, se va construyendo en la interacción del sujeto que investiga con el objeto que es investigado. En el caso del presente estudio, de la investigadora con la docente, con los alumnos y las alumnas así como con los objetos del espacio del aula y su entorno más inmediato, la escuela.

Esta investigación al asumir el trabajo que se desarrolló básicamente con un grupo de estudiantes y en un contexto determinado, donde se logra un análisis cualitativo y profundizar en la labor que se llevó a cabo, se enmarca dentro de los métodos de investigación 
con estudio de casos. Stake (1998) presenta una visión de los estudios de caso que parte de los métodos de investigación naturalistas, holísticos, etnográficos, fenomenológicos y biográficos. El mismo autor, señala que el "caso" es "único, algo específico, complejo, en funcionamiento, por lo que el investigador se debe concentrar en él para lograr comprenderlo" (Stake, 1998:16-17). El autor antes citado considera que la misión que tiene el estudio de casos es la particularización y no la generalización, por lo que el investigador toma un caso en particular y llega a conocerlo bien, a profundidad y lo hace no para establecer relación con otros casos, ver en qué se parece y en qué se diferencia, sino por el contrario, al ser el caso único, debe tratarlo como tal y de esa manera logrará comprenderlo en su más enriquecedora dimensión.

El estudio de casos, de acuerdo con Van Dalen y Meyer (1979) si se quiere tiene un alcance más limitado pero es más exhaustivo que otros tipos de investigación y concede mayor importancia a aspectos de carácter cualitativo, por lo que los aportes de ese tipo de estudios son muy valiosos en una investigación como ésta.

Debido a las características de la investigación realizada como lo fue el captar el fenómeno de lo observado en un aula de Primer año, se recurrió a elementos de la metodología de la investigación fenomenológica. Esta ciencia estudia los fenómenos tal y como son experimentados, vividos y percibidos por el ser humano (Martínez, 1989), en el caso de la presente investigación, por la investigadora.

Martínez considera que las "realidades cuya naturaleza y estructura peculiar sólo pueden ser captadas desde el marco de referencia interna del sujeto que las vive y las experimenta, exigen ser estudiadas desde el método fenomenológico" (Martínez, 1989:167). Para el autor antes citado, la realidad que se está estudiando no es una realidad objetiva, externa, igual para todos tal y como suele considerarse desde el paradigma positivista, le agregaría yo, sino que por el contrario es una realidad "cuya esencia depende del modo en que es vivi- da y percibida por el sujeto, una realidad interna y personal, única y propia de cada ser humano" (Martínez, 1989:167).

Uno de los objetivos al recurrir a elementos de la fenomenología fue el de describir el fenómeno en estudio, los procesos de lectura y escritura tal y como se llevaron a cabo en un Primer año de I Ciclo, de la forma más completa y detallada posible, como se plasmó en los diversos tipos de notas, para de esa manera, poder reflejar la realidad del aula donde se desarrollaba la investigación y reflexionar sobre ella y desde ella, así como lograr captar los significados que los sujetos de esos procesos iban generando en ese contexto. En esa aula se fue conformando poco a poco un grupo de niños y niñas. Algunos de los niños y algunas de las niñas se conocían desde el jardín infantil, otros y otras por el contrario, se encontraron por vez primera en ese espacio donde guiados por la maestra, fueron tejiendo su propia historia al desarrollar, poco a poco, cual los hilos de una madeja, los procesos de lectura y escritura. Esa historia fue construida mediante la interacción de los actores y de las actoras de esos procesos al realizar diversas actividades y utilizar materiales variados. Motivada por el deseo de captar los fenómenos que acontecían en ese espacio fue que recurrí a la observación directa o participante, a tomar mis notas crudas y luego elaborar con base en ellas, las notas cocidas, al recoger datos e información de diversas fuentes: maestra, niños y niñas, documentos, materiales, investigadoras externas al espacio que constituía en ese momento el núcleo del estudio; el aula de ese Primer año. Asimismo recurrí a técnicas como la entrevista coloquial, la conversación, el diálogo tal y como lo sugiere Martínez, con el fin de obtener la mayor información posible y profundizar los datos obtenidos.

El observar el aula como un fenómeno único por la naturaleza de las relaciones que ahí se generaron me permitió desde mis experiencias, describir, interpretar y comprender mejor lo que aconteció en ese espacio durante las observaciones que hice en él y de él. 
Otro de los métodos a los que recurrí durante la investigación realizada fue al de la hermeneútica. Dilthey, citado por Martínez, la definió como "el proceso por medio del cual conocemos la vida psíquica con la ayuda de signos sensibles que son su manifestación" (Martínez, 1989:119). En consecuencia con lo anterior, la hermeneútica tiene como misión el describir así como el interpretar de la mejor manera posible los significados de las cosas, de las palabras, de los escritos, de los textos así como los gestos de las personas dentro del contexto en el cual tienen lugar. Es por ello que de acuerdo con Martínez (1989) la hermeneútica debe de cristalizar "los trozos de vida" o "eventos existenciales" que tienen lugar en un espacio y momento determinado para lo cual hoy el investigador o la investigadora pueden recurrir a medios como los que la electrónica y la tecnología ponen a nuestro alcance. Mediante ellos es posible captar el movimiento de las cosas, los gestos, las voces así como los otros sonidos que producen para recrearlos y de esa manera, tratar de captar los significados que encierran.

De acuerdo con Martínez (1989), Dilthey, realiza un gran aporte al desarrollo de las ciencias humanas, al plantear la idea de que no sólo los textos escritos, sino toda la expresión de la vida humana, es objeto natural de la interpretación hermeneútica. Lo anterior debido a que las operaciones mentales que realizan los individuos al tratar de obtener el significado de los textos son las mismas que llevan a cabo cuando conocen otra realidad humana. Por consiguiente la hermeneútica, de acuerdo con Dilthey, permite conocer el significado del comportamiento de los individuos, las formas no verbales de la conducta (a las que se recurre muchas veces en el trabajo de aula), a los diversos sistemas culturales (el aula es uno de ellos debido a las relaciones que ahí se dan así como a las características de los sujetos que conforman ese núcleo, son sujetos históricos), a las organizaciones sociales así como a los sistemas conceptuales o filosóficos (Martínez, 1989).

Dentro de la hermeneútica cobra vital importancia el contexto en el cual se desarro- llan las acciones objeto de estudio. Asimismo ese contexto se analiza de igual forma que un texto escrito, pues al llevar a cabo ambos análisis, la persona que lo realiza recurre a las mismas operaciones de pensamiento como lo son el establecer semejanzas y diferencias entre sus vivencias y lo nuevo, realizar comparaciones, interpretaciones, entre otras operaciones mentales. De acuerdo con lo anterior, la investigación se lleva a cabo tomando en cuenta no sólo el hecho en particular sino la relación de éste con el contexto social en el que está inmerso. Debido a lo anterior, en la investigación llevada a cabo se recurrió a la dialéctica al proceder del todo a las partes y de las partes al todo como lo fue ver el aula dentro de la escuela, la escuela en la comunidad y su relación con el sistema educativo. Asimismo cómo la política educativa incide en las acciones que se llevan a cabo en la escuela y en el contexto de aprendizaje como lo es el aula. Lo anterior con el fin de establecer las relaciones entre lo singular y lo general y entre lo global y lo particular. En este sentido es importante destacar el apoyo que en la hermeneútica encontré durante el desarrollo de la investigación pues pude interesarme por llevar a cabo una lectura cuidadosa del contexto en que se encuentra la escuela y el aula, por comprender e interpretar los significados de los gestos tanto de la maestra como de los niños y de las niñas. Asimismo comprender que las actividades se realizan en un tiempo y lugar determinado lo que les da un carácter histórico e irrepetible por lo tanto esas acciones aunque queramos, no se pueden llevar a cabo nuevamente tal y como ocurrieron.

Otro aspecto importante por destacar desde la aplicación de la hermeneútica a la presente investigación, es el poder establecer la secuencia entre todas las actividades que se desarrollaron en el aula pues ellas condujeron a un todo que es el aprendizaje permanente en los escolares y las escolares. Así como la relación del aula con su contexto; la escuela y de ésta con el entorno social en el que se encuentra. Por otro lado, me hizo ver la necesidad de que las acciones del aula deben estar 
encaminadas hacia la contextualización de los contenidos y temas que se desarrollen pues éstas se dan como parte de un sistema de relaciones: aula, escuela, contexto social y no en forma aislada. Debido a lo anterior, el trabajo del aula se debe de desarrollar considerando las experiencias previas de los estudiantes y de las estudiantes para que les sean realmente significativos.

El método fenomenológico de acuerdo con Martínez (1989) centra su atención en el fenómeno, en la forma cómo éste se presenta, en las realidades vivenciadas por los sujetos y respeta plenamente la relación de las personas, mediante sus propias vivencias, con lo observado. Por ser esa experiencia de carácter personal y de acuerdo con el autor citado, no habría ningún motivo para dudar de las vivencias que las personas dan a conocer. El mismo autor (Martínez:1989) al referirse al método hermeneútico considera que éste permite "introducirse en el contenido y en la dinámica de la persona estudiada" y busca estructurar una interpretación coherente del todo. Ambos métodos, aportaron elementos para llevar a cabo un estudio analítico, interpretativo, sociohistórico pues los acontecimientos se dan en un aquí y un ahora.

La investigación llevada a cabo consta de dos partes: la primera es una investigación de carácter etnográfico que recibió aportes del método fenomenológico y el hermeneútico y la segunda, una investigación acción. Es importante destacar el hecho de que ésta fue una situación que emergió en la construcción del diseño debido a que durante el desarrollo del estudio, la docente me planteó una serie de necesidades lo que me llevó a generar acciones de actualización tanto a esa docente como a los maestros y a las maestras de la institución.

Es importante señalar el hecho de que a partir de la investigación realizada, se delinearán algunos elementos por considerar en el diseño de una propuesta de actualización en los campos de la lectura y de la escritura para docentes en servicio y sugerencias para la formación de maestros y maestras de Educación General Básica en esos aspectos.
El hecho de que la presente investigación se llevó a cabo en el ámbito escolar, permitió que el estudio se centrara en descubrir lo que allí aconteció cotidianamente en relación con la atención de los procesos de lectura y escritura. Los datos que recogí fueron organizados en las categorías que surgieron a partir del análisis de la información y que mencionaré más adelante, de esa forma, pude interpretar y comprender lo que sucedió durante los meses que visité ese nicho tal y como lo citan Goetz y Le Compte llamado "aula" (Goetz y Le Compte, 1988:14). La información fue recolectada en ese contexto, donde interactuaron la maestra y los niños y las niñas. Asimismo tuve que considerar otros sujetos como los investigadores e investigadoras externas al trabajo del aula, el director, las maestras y los maestros de otros grupos así como de asignaturas especiales. La segunda parte de la investigación consistió en una investigación acción. Esa situación se debió a que la docente, en diferentes momentos del trabajo de aula y con base en la reflexión de su trabajo en el aula, recurrió a mí en la búsqueda de apoyo para el desarrollo de su labor así como para la organización de actividades de enriquecimiento no sólo de los niños y niñas de otros años, como lo fue el Taller sobre Literatura Infantil con los alumnos y alumnas del II Ciclo, de los docentes y las docentes de la institución, de los directores y directoras de las diferentes instituciones educativas que conforman el Distrito primero de la Provincia de Heredia. Entre ellos y ellas se encontraban directores y directoras de Educación preescolar, de I y II Ciclos, Enseñanza Media y Técnica. Las necesidades planteadas por la docente se enmarcaron dentro de temas como la metodología de la lectura y de la escritura, la evaluación auténtica así como la atención a niños y niñas con dificultades en el aprendizaje, aspectos por considerar en procesos de formación y de actualización de los docentes y de las docentes.

En cuanto a la investigación acción y de acuerdo con Fernández (1995), lo esencial de ese tipo de investigación puede resumirse en los siguientes puntos: 
- $\quad$ Los sujetos implicados profesionalmente en determinadas tareas realizan su labor con el asesoramiento de las personas externas requeridas.

- Se realiza un análisis de la propia práctica.

- Con base en ésta se formula un plan controlado de intervención de la práctica analizada.

- El trabajo producto de la intervención se evalúa posteriormente.

- $\quad$ Con base en los resultados, se inicia un ciclo de trabajo como el que se llevó a cabo. Esto plantea un mejoramiento constante de la actividad en el aula (Fernández, 1995:144).

Al elaborar la propuesta metodológica para la investigación que llevé a cabo me fue necesario recordar que la investigación educativa, como investigación aplicada, está condicionada por una finalidad prioritaria que es apoyar los procesos de reflexión y crítica con el fin de mejorar la calidad de los procesos de enseñanza-aprendizaje (Goetz y LeCompte, 1988).

El mejoramiento de la calidad de la enseñanza debe unir por ello teoría y práctica, o lo que es igual, hacer conscientes a los profesores y a las profesoras de las teorías que guían su labor, sobre todo antes de que ésta tenga lugar y no únicamente después, una vez que los alumnos y las alumnas sufrieron las consecuencias que, muchas veces, podrían ser evitadas (Goetz y LeCompte, 1988).

\section{Herramientas utilizadas para adentrarme en la cultura del aula}

Dentro de los instrumentos utilizados para recabar los datos, puedo mencionar los siguientes:

1. El diario de la maestra.

2. Fotografías donde se destacan momentos del trabajo de la maestra con los niños y las niñas, de los niños y niñas en forma individual y grupal y de algunos de los materiales que han trabajado a esta fecha.

3. La observación participante y la no participante.

4. Entrevistas a la maestra, niños, niñas, director, y otras maestras de la institución. Entre las entrevistas utilizadas están la entrevista en profundidad y la entrevista estructurada.

5. Análisis de documentos.

6. Conversaciones informales con el señor director de la institución y con la bibliotecaria.

Asimismo realicé diversas actividades, dentro de las que puedo mencionar las siguientes:

1. Toma de vídeos.

2. Grabaciones de conversaciones con la docente.

3. Participación de observadores externos.

4. Realización de un Taller de Expresión Plástica con los niños y niñas del grupo donde llevaba a cabo mi investigación.

\section{Herramientas y procesos que facilitaron mi labor}

En el desarrollo de la investigación, recurrí a la observación participante, no participante y a la entrevista en profundidad. Taylor y Bogdan (1996) al referirse a la observación participante consideran que trabajar con este instrumento requiere de tres actividades principales, éstas se describen a continuación.

Es importante señalar que en este tipo de investigación, los observadores y las observadoras participantes entran en el campo donde se va a realizar el estudio con la esperanza de establecer una relación abierta, fluida con los informantes y las informantes. Lo deseable es que lleguen a formar parte de ese contexto social de tal manera que no se sientan como extraños en el lugar sino por el contrario, Como parte de ese entorno. Es durante ese período cuando el observador participante o la 
observadora participante debe ganarse la confianza de los sujetos que va a observar, de ahí que la recolección de datos pase a ser secundaria.

Igualmente, es necesario destacar que la negociación de la observación debe ser constante a fin de respetar el espacio y las actividades que los sujetos realizan. De acuerdo con Taylor y Bogdan establecer "rapport" con los informantes y las informantes es la meta principal de todo investigador e investigadora de campo, lo que hace necesario tener presente esa negociación (Taylor y Bogdan, 1996).

En cuanto al papel de los informantes claves, de acuerdo con los mismos autores los definen como quienes "apadrinan al investigador en el escenario y son sus fuentes primarias de información" (Taylor y Bogdan, 1996:61). Son personas respetadas y conocedoras de los asuntos por investigar y se convierten en los mejores amigos y amigas del investigador en el campo.

Al utilizar la observación participante, el investigador debe considerar que ésta depende de un registro de notas de campo lo más completo posible. Estas deben ser precisas y detalladas para lo cual debe tener presente la regla que establece lo siguiente "si no está escrito no sucedió nunca" (Taylor y Bogdan, 1996:75).

Es importante mencionar que en el desarrollo del presente estudio además de las observaciones realizadas por la investigadora se ha recurrido a la participación de un observador y cuatro observadoras externas tal y como lo mencioné anteriormente. La presencia tanto de esas investigadoras como del especialista en Artes Plásticas quien llevó a cabo el Taller con los niños y niñas en el grupo donde realicé el estudio, fue sumamente importante ya que mediante sus observaciones, brindaron elementos que permitieron validar los hallazgos del estudio, favoreciendo de esa manera la aplicación de la "triangulación" a la información recabada lo que permitió validar los datos que poseía, desde diversas fuentes. Asimismo sus comentarios permitieron orien- tar la observación hacia aspectos de la práctica cotidiana que muchas veces por considerarse sumamente obvios merecen mayor atención por parte del investigador.

Con respecto a la entrevista en profundidad, es importante destacar el hecho de que contrariamente a la entrevista estructurada, éstas son flexibles y dinámicas. Esas entrevistas consisten en reiterados encuentros cara a cara entre investigador e informantes, encuentros éstos dirigidos hacia la comprensión de las perspectivas que tienen los informantes respecto de sus vidas, experiencias o situaciones, tal y como lo expresan con sus propias palabras (Taylor y Bogdan, 1996). Este tipo de entrevista sigue el modelo de una conversación "entre iguales" y por lo tanto no constituye un intercambio formal de preguntas y respuestas, razón por la cual su empleo resulta un valioso recurso cuyo fin es recabar información de carácter cualitativo, más espontánea y emergente en las actividades que se realizan.

Con el propósito de guiar las entrevistas iniciales, el investigador puede recurrir a las preguntas descriptivas, los relatos solicitados, la entrevista con cuaderno de bitácora y los documentos personales. Estas deben darse en un clima donde de acuerdo con Taylor y Bogdan se debe tener presente lo siguiente:

- No abrir juicio.

- Permitir que la gente hable.

- Prestar atención.

- Ser amable (Taylor y Bogdan, 1996: 121-122).

Con base en la utilización de los instrumentos anteriores el trabajo de campo relacionado con la presente investigación, se realizó tomando en cuenta los siguientes aspectos:

\section{Mi acercamiento a la institución educativa}

Esta investigación dio inicio en el mes de febrero de 1999, con el comienzo del año lectivo. La primera reunión con el señor Director, 
fue llevada a cabo el miércoles 10 de ese mes en horas de la mañana pues cuando fui a visitarle en el mes de diciembre del año anterior, me sugirió que volviera al iniciar el curso lectivo del año siguiente debido a que a esa fecha ya tendría los maestros y las maestras asignados a cada grupo. En esa oportunidad le presenté el proyecto por desarrollar y una carta donde le solicitaba formalmente un grupo de primer año para realizar la investigación. Conversamos acerca de las ventajas que en una propuesta de formación y actualización de docentes, tiene la información que se logre recabar desde el trabajo de aula. En esa ocasión el Director sugirió trabajar con la maestra coordinadora de los primeros años por lo que al día siguiente volví a la institución para solicitarle a ella una reunión. En esa reunión junto con la docente llegamos a un acuerdo en relación con los días en que visitaría al grupo.

\section{Mis observaciones en el primer año}

Tanto la maestra como yo, consideramos al inicio del curso lectivo que el lunes y el jueves eran los días más convenientes para las actividades en relación con la presente investigación. Seleccionamos el lunes para tener la oportunidad de ver el trabajo que realizaban en horas de la mañana además, en el segundo período los niños recibían música lo que nos permitió a la docente y a mí, intercambiar opiniones respecto al trabajo, poder realizar mis entrevistas, revisar los cuadernos de los niños y de las niñas y dar seguimiento a su progreso. Asimismo apoyar a la docente con algunas lecturas e ideas respecto al trabajo que llevaba a cabo, ideas sobre evaluación dentro de un paradigma cualitativo, sobre cómo poder orientar a los niños y niñas en sus procesos de construcción de la lectura y escritura.

Debido a la necesidad de tener un distanciamiento del escenario objeto de estudio tal y como lo sugieren Taylor y Bogdan (1996), suspendí mis observaciones en junio y julio por lo que me reincorporé en agosto. Desde ese mes continué visitando el grupo hasta octubre que concluí con el desarrollo del Taller de Expresión Plástica. Durante los meses que visité el grupo, realicé aproximadamente tres reuniones para la inserción tanto en el centro educativo como en el grupo, veintisiete observaciones, dos talleres con los niños, una reunión con los directores de las instituciones educativas; desde el preescolar hasta la enseñanza diversificada, del Circuito Primero de la Provincia de Heredia e hice posible la participación de académicos de la Universidad Nacional en cuatro talleres donde se abordaron temáticas con base en los intereses y necesidades de los maestros y las maestras de la institución.

Las observaciones realizadas permitieron reflexionar aún más sobre la importancia del tema investigado. Esa reflexión era consignada en las crónicas correspondientes a esas observaciones. La elaboración de esas crónicas o "notas cocidas" surgió de las "notas crudas" que iba tomando durante mi participación en las actividades de observación. En las crónicas realicé una descripción cuidadosa de lo observado: consignando el día en que se realizó la observación, los objetivos de la actividad o actividades. Seguidamente elaboré una descripción detallada de lo observado destacando la participación de la maestra así como de los niños y niñas por ser los principales protagonistas y las principales protagonistas del estudio que se llevó a cabo. La crónica la concluía, generalmente, con un comentario o reflexión personal; momento que considero sumamente importante porque me permitió meditar acerca de lo observado, confrontarlo con mi posición teórica y a la vez, elaborar mis ideas al respecto. Aprovechaba ese momento para relacionar el trabajo observado con las fuentes teóricas en que se sustentaba así como con planteamientos más innovadores respecto a la atención de los procesos de lectura y escritura en los diferentes momentos de su desarrollo.

Es durante ese momento, en la reflexión personal, donde tiene lugar un "encuentro" con las preguntas de investigación planteadas al inicio del estudio y que dieron pautas

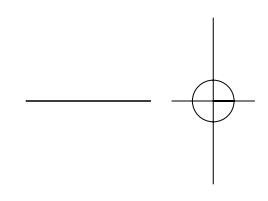


al desarrollo de la investigación. Asimismo realicé la revisión de esas preguntas, eliminando las que no eran pertinentes, generando otras y tratando de conformar las respuestas a las interrogantes que forman parte del diseño. Esas respuestas están respaldadas por las observaciones, fotografías, vídeos y otros materiales lo que permitió validar la información recabada.

Creo que ese momento de reflexión me permitió ese "encuentro", que considero único y por lo tanto sumamente importante, con mis ideas, con mi elaboración teórica acerca de la educación y los procesos de lectura y escritura. Asimismo ese espacio constituyó una valiosa oportunidad para ubicar lo observado en el contexto de la investigación.

\section{Categorias y preguntas que orientaron el análisis de la información}

Tal y como lo mencioné en párrafos anteriores, el problema delimitado y los objetivos propuestos me llevaron al planteamiento de seis categorías y preguntas que orientaron la investigación realizada.

La organización de las preguntas en esas categorías, orientó la elaboración de los instrumentos por utilizar durante el desarrollo de la investigación. Lo anterior me permitió ir modificando, eliminando o generando las preguntas que inicialmente plantee para guiar esta investigación, labor realizada a la luz de ese proceso. Esa situación me hizo posible eliminar algunas de esas preguntas o bien incorporar otras que resultaran más significativas en el trabajo que llevé a cabo. Las categorías construidas son las siguientes:

I CATEGORÍA: ¿Cuáles son las características personales y las calidades académicas de la docente?

II CATEGORÍA: ¿Cuáles son los aspectos más relevantes que presenta la docente en relación con su desarrollo profesional?

III CATEGORÍA: ¿Qué elementos considera la docente en la planificación de su labor?
IV CATEGORÍA: ¿ Dónde está ubicado el centro educativo? ¿Cuáles han sido y son las características más importantes de esa institución escolar?

V CATEGORÍA: ¿Qué características de los niños y de las niñas inciden en sus procesos de aprendizaje?

VI CATEGORÍA: ¿Cómo orienta la docente los procesos de enseñanza y aprendizaje en el aula?

Asimismo esas categorías y sus preguntas facilitaron una "lectura" de los fenómenos que se generaron en el aula durante mis períodos de observación. La descripción de las situaciones de aprendizaje, su análisis e interpretación así como los hallazgos que encontré en la labor del aula, me permitieron la construcción de los significados.

\section{Un camino que emerge}

\section{Análisis e interpretación de la información}

El análisis de la información recabada durante mis visitas al aula, me permitió develar aspectos de la práctica pedagógica que permiten o impiden a la maestra innovar su labor. El análisis de esa información forma parte de este apartado y para realizar esa labor me basé entre otros autores, en Shagoury y Miller (1993).

Para esas investigadoras, el análisis de la información es un proceso que permite ordenar los datos obtenidos, estructurarlos y darles sentido, así como buscar el significado o los significados que subyacen en ellos.

En la búsqueda por explicarse el material recolectado, de acuerdo con las autoras mencionadas, los maestros y las maestras establecen un diálogo con esos materiales, los cuestionan, les encuentran nuevos significados, ritmos y rimas a lo escrito. Ese análisis de acuerdo con Shagoury y Miller (1993) es fascinante, puesto que le permite al investigador, encontrarle sentido a la información y elaborar teorías respecto a lo que 
se está investigando. Es con base en el análisis de los datos que se van definiendo las categorías con las que se va a trabajar pues de ahí emerge los aspectos que se reiteran y los que llamaron la atención.

La información que logré obtener durante mis visitas a la institución, realizadas durante los meses comprendidos entre febrero y noviembre de 1999 en ese Primer año, fue levantada inicialmente en "notas crudas". Estas son las notas que tomé en el momento de la observación, de la entrevista, de la conversación, en el trabajo de campo. Luego esas notas las organicé en las crónicas o "notas cocidas" (Shagoury y Miller, 1993), las que fueron objeto de un cuidadoso análisis así como de su interpretación.

Para Shagoury y Miller (1993), aprender a tomar notas es un proceso que nunca termina pues siempre se está en la búsqueda de las mejores formas de hacerlo. Fue precisamente con base en la idea anterior que las crónicas levantadas requirieron de varias lecturas para poder ver si en ellas representaba mi forma de ver el aula así como de los procesos que en relación con la lectura y escritura en ella se generaban.

Para facilitar el proceso de análisis de las notas cocidas, asigné un color a cada una de las categorías. La utilización de diferentes colores me permitió ir identificando, en las notas cocidas, la información correspondiente a cada una de las categorías lo que me ayudó a relacionar la información obtenida con las preguntas que orientaron el estudio y ubicarla en la categoría correspondiente. Fue así como asigné un color a cada categoría tal y como puede observarse seguidamente:

\section{CATEGORÍA}

COLOR QUE LA IDENTIFICA

$\begin{array}{ll}\text { Docente } & \text { anaranjado } \\ \text { Planificación del trabajo } & \text { azul } \\ \text { Centro educativo } & \text { rojo } \\ \text { Niños } & \text { verde } \\ \text { Entorno } & \text { morado } \\ \text { Trabajo de aula } & \text { café }\end{array}$

Durante el análisis de las crónicas o "notas cocidas", fui consignando las notas de carácter teórico, metodológico, personal y de campo (Shagoury y Miller, 1993). La labor realizada me permitió, mediante un proceso constante de reflexión, sustentar teóricamente las observaciones, comentarios, entrevistas que llevé a cabo durante la investigación. Asimismo recurrí a algunas de las estrategias que Goetz y LeCompte (1998) sugieren para el análisis de la información, entre ellas la inducción analítica, la comparación como una actividad constante, la confrontación de la información con las categorías que orientan el estudio y la enumeración de los elementos que se iban descubriendo.

En ese proceso elaboré diferentes matrices, lo que me permitió visualizar las variadas formas en que se pueden organizar los datos obtenidos y diseñar los modelos que respondieran a la información recabada. Fue en las obras de Maxwell (1996), Shagoury y Miller (1993), Del Rincón y colaboradores (1995), Taylor y Bogdan (1996) y Valles (1997), especialmente en las que me basé para construir y reconstruir las dos matrices (estas se presentan en el presente artículo) que utilicé en la labor de organización y análisis de la información recabada durante 
los meses que comprendió el estudio, información que se encontraba en las crónicas o "notas cocidas". Como puede observarse en las matrices que elaboré, éstas responden a las características de los datos así como de las situaciones de aprendizaje descritas en las crónicas que como ya mencioné, constituyen el material básico en este proceso.

Matriz $\mathrm{N}^{\circ} 1$

Acercamiento a los elementos de la investigación

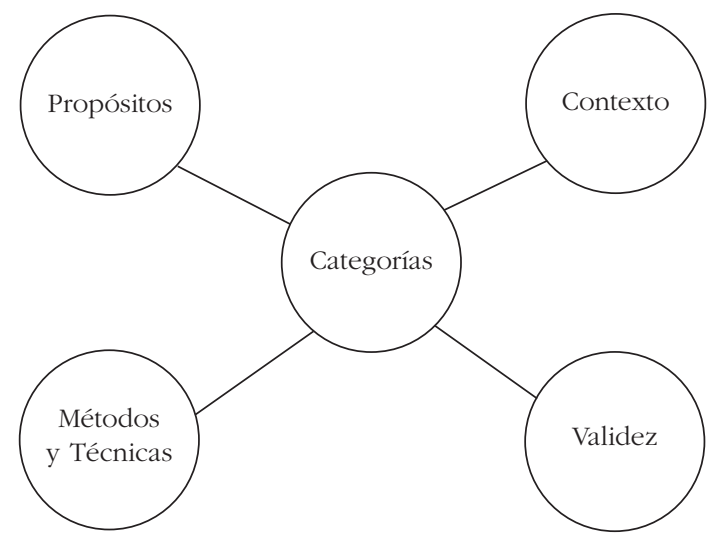

Fuente: Adaptado de la obra de Maxwell, Joseph A. (1996). Qualitative Research Design and Interactive Approach. P. 62.

En esas matrices, a manera de párrafo, presento la información de acuerdo con el problema que deseaba investigar de ahí que aparezcan la preguntas de investigación numeradas del uno al seis, los objetivos por alcanzar, elementos del contexto, tanto de carácter teórico como histórico, educativo, social, cultural, económico en que se encuentra la institución donde se llevó a cabo el presente estudio, las preguntas que orientaron la investigación y las notas que respaldan los eventos observados. Esas notas las elaboré con base en la clasificación de Shagoury y Miller (1993) y que como mencioné se clasifican en notas de campo, de carácter teórico, metodológico y personales. Estas fueron surgiendo a lo largo de la investigación pues tienen diferentes características. Seguidamente me referiré a dos de esas notas pues considero que su elaboración fue básica en el proceso de análisis e interpretación de la información: teóricas y personales. Estas se constituyeron en fuentes muy importantes para respaldar mis observaciones, entrevistas, conversaciones, fotografías y videos realizados. En cuanto a las notas teóricas éstas me permitieron relacionar lo observado en el aula con notas bibliográficas para fundamentar lo que acontecía en ese espacio. Lo anterior me llevó a una búsqueda constante de literatura, de fuentes, a una selección cuidadosa de textos, citas en las que pudiera apoyar lo observado. Con respecto a las notas personales, considero que éstas son sumamente importantes pues en el momento de su elaboración el investigador o la investigadora se encuentran con sus teorías, creencias y metáforas frente a lo observado. Esto, en mi caso, me permitió reflexionar y replantearme las ideas mediante las vivencias que estaba teniendo en ese momento, paso sumamente importante en la construcción de la teoría que emerge del análisis e interpretación de los datos que poseo. Ejemplo de organización de los datos en la Matriz $\mathrm{N}^{\circ} 2$ 
Matriz No 2

APROXIMACIONES A LA LABOR EN EL AULA

15 de febrero

\begin{tabular}{|c|c|c|c|c|c|}
\hline $\begin{array}{c}\text { Número de } \\
\text { veces que se } \\
\text { presenta }\end{array}$ & $\begin{array}{l}\text { Preguntas de } \\
\text { investigación }\end{array}$ & Participantes & $\begin{array}{l}\text { Eventos observa- } \\
\text { dos: (principio, } \\
\text { desarrollo, fin) }\end{array}$ & $\begin{array}{l}\text { Notas (persona- } \\
\text { les, teóricas, } \\
\text { metodológicas } \\
\text { y de campo) }\end{array}$ & $\begin{array}{l}\text { Procesos: } \\
\text { Enseñanza- } \\
\text { Aprendizaje }\end{array}$ \\
\hline & $\begin{array}{l}\text { III ÁREA: ¿Qué } \\
\text { elementos consi- } \\
\text { dera la docente } \\
\text { en la planifica- } \\
\text { ción de su labor? } \\
\text { a. El conoci- } \\
\text { miento del pro- } \\
\text { grama de Primer } \\
\text { Grado y del I Ci- } \\
\text { clo. La forma en } \\
\text { que planea su } \\
\text { trabajo en rela- } \\
\text { ción con los pro- } \\
\text { cesos de lectura } \\
\text { y escritura. Las } \\
\text { adecuaciones del } \\
\text { currículum oficial } \\
\text { a las necesidades } \\
\text { e intereses de los } \\
\text { niños y a las ca- } \\
\text { racterísticas del } \\
\text { entorno. En este } \\
\text { sentido, se de- } \\
\text { ben indicar en } \\
\text { qué consisten es- } \\
\text { tas adecuaciones. }\end{array}$ & $\begin{array}{l}\text { Maestra y niños } \\
\text { y niñas. }\end{array}$ & $\begin{array}{l}\text { La maestra inició } \\
\text { el trabajo de Pri- } \\
\text { mer grado con la } \\
\text { etapa de Apresta- } \\
\text { miento. }\end{array}$ & $\begin{array}{l}\text { Nota personal } \\
\text { Al comienzo de } \\
\text { la entrevista que } \\
\text { tuve con la } \\
\text { maestra esa ma- } \\
\text { ñana, me mani- } \\
\text { festó la necesi- } \\
\text { dad de utilizar } \\
\text { "métodos no tra- } \\
\text { dicionales" en el } \\
\text { trabajo con los } \\
\text { niños para poder } \\
\text { corresponder a } \\
\text { sus expectativas, } \\
\text { sin embargo, en } \\
\text { el tercer período } \\
\text { de esa mañana, } \\
\text { el trabajo con- } \\
\text { sistió en realizar } \\
\text { actividades que } \\
\text { podríamos ubicar } \\
\text { en el paradigma } \\
\text { tradicional. } \\
\text { Nota Teórica } \\
\text { "Los niños pue- } \\
\text { den adquirir la } \\
\text { lectura y la escri- } \\
\text { tura con los tex- } \\
\text { tos que ellos son } \\
\text { capaces de pro- } \\
\text { ducir y no supe- } \\
\text { ditarlos al "libro } \\
\text { de texto" que co- } \\
\text { mo sabemos no } \\
\text { satisface los inte- } \\
\text { reses y necesida- } \\
\text { des de los alum- } \\
\text { nos" "La práctica } \\
\text { pedagógica que } \\
\text { concibe al niño } \\
\text { como productor } \\
\text { de textos es to- } \\
\text { mada en cuenta } \\
\text { por el constructi- } \\
\text { vismo. }\end{array}$ & $\begin{array}{l}\text { Proceso ense- } \\
\text { ñanza apren- } \\
\text { dizaje: } \\
\text { Ese día la maes- } \\
\text { tra había pla- } \\
\text { neado el trabajo } \\
\text { con un libro } \\
\text { que contiene } \\
\text { actividades de } \\
\text { aprestamiento. } \\
\text { Para dar inicio a } \\
\text { la lección, la } \\
\text { maestra solicitó } \\
\text { a los niños que } \\
\text { se sentaran cada } \\
\text { uno en su lugar } \\
\text { y prestaran } \\
\text { atención. } \\
\text { En la produc- } \\
\text { ción de textos } \\
\text { por los niños } \\
\text { conviene con- } \\
\text { siderar las ideas } \\
\text { de Freinet al } \\
\text { respecto. } \\
\text { En su obra } \\
\text { Freinet destata- } \\
\text { ca la importan- } \\
\text { cia de la escri- } \\
\text { tura por parte } \\
\text { de los niños. } \\
\text { Freinet propo- } \\
\text { ne reali-zar } \\
\text { ejercicios de } \\
\text { escritura que } \\
\text { permitan al ni- } \\
\text { ño famiariliari- } \\
\text { zarse con la } \\
\text { lengua escrita, } \\
\text { y a la vez, esta- } \\
\text { blecer la rela- } \\
\text { ción entre el } \\
\text { pensamiento y } \\
\text { el grafismo. }\end{array}$ \\
\hline
\end{tabular}

Fuente: Registro de las notas levantadas en el desarrollo de la investigación. 
Es necesario destacar el hecho de que las "notas cocidas" se organizaron en la matriz número dos con una secuencia temporal e histórica, haciendo uso de los aportes de la hermeneútica, pues si bien es cierto que las primeras notas habían sido elaboradas de acuerdo con las pautas que orientaban las observaciones, fue en el momento preciso de ordenar los datos en la matriz, donde surgió la necesidad de estructurar esa información de acuerdo con el diseño con que la docente observada, orientaba los procesos de lectura y escritura. La decisión anterior permitió elaborar "una guía de la historia" tal y como lo sugieren Taylor y Bogdan (1996:166) pues el diseño de los procesos de aula, se convirtió en la hebra que permitió darle una perspectiva histórica al estudio e hilar en torno a esos procesos, los otros aspectos presentes en la observación como por ejemplo la utilización del método de juego, el período de círculo, el desarrollo de actividades lúdicas, entre otros. Lo anterior me permitió orientar el análisis y la interpretación de la información de tal manera que se viera la evolución que los niños y las niñas, como protagonistas de la investigación, iban teniendo en relación con esos procesos. Por lo tanto, la organización de los datos se realizó a partir de la información recabada desde los momentos iniciales que los niños y las niñas viven con respecto a la lectura y la escritura como lo es la continuación de la etapa del aprestamiento. Con esta etapa se inició la labor en ese primer año así como la secuencia que la docente brindó a esos procesos a lo largo del curso lectivo. En ese desarrollo se apreció la adquisición del código escrito que puede considerarse como un aspecto histórico en la vida de los hombres y de las mujeres debido a que como sabemos, la alfabetización abre un horizonte inigualable a cada ser humano otorgándole las herramientas necesarias para desenvolverse en el medio donde se encuentre. De acuerdo con lo anterior Bettelheim y Zelan (1986) consideran que para los niños y las niñas la forma en que en que se les enseña a leer es de vital importancia pues el aprendizaje de la lectura determinará en ellos y en ellas su opinión no solo en torno al aprendizaje en general sino también al concepto de sí mismo como aprendiz y como persona.

Lo anterior lleva a pensar en el aporte de Freire (1997) al destacar el hecho de que los procesos de lectura y escritura sean éstos para alfabetizar o post alfabetizar, deben tomar como punto de partida la visión del mundo que los hombres y las mujeres poseen, es decir en palabras del educador brasileño, su "capital cultural" pues es de esa forma que esos procesos les son significativos.

Una vez que organicé y analicé los datos en la matriz número 2 , actividades que tomaron aproximadamente tres meses de este proceso debido a que fue una labor cuidadosamente llevada y que me permitió visualizar tanto la totalidad de la investigación como los diversos aspectos que la conforman, incorporar las notas teóricas, plantearme nuevas interrogantes, procedí a la lectura nuevamente de la información pues tal y como lo señalé en párrafos anteriores, en ese esquema aparece la información organizada de acuerdo con las seis columnas que integran esa matriz.

La organización de la información en la matriz número dos me llevó a un proceso de selección de la información por lo que algunas de las notas cocidas no se incorporaron en ese esquema. Por consiguiente, esa información forma parte únicamente de ese tipo de notas. La selección de notas la llevé a cabo debido a las características que iba adquiriendo el proceso mismo pues conforme avanzaba en la organización de los datos en la matriz, era necesario elegir las notas que se consideraban más relevantes con respecto a los objetivos y a las preguntas de investigación que orientaban el presente estudio. Asimismo la incorporación de las diferentes notas siguiendo la clasificación de Shagoury y Miller (1993) como actividad simultánea a la selección de la información, me llevó a una comprensión mayor del proceso vivido en el aula así como a una relación de lo observado con los aspectos teóricos que le dan fundamento.

Realizada la lectura global de la información que presento en la Matriz $\mathrm{N}^{\circ} 2$, procedí al análisis e interpretación de la misma

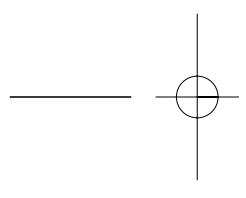


de acuerdo con las preguntas de investigación. Ese análisis e interpretación se ubica en cinco grandes categorías. Por consiguiente las categorías que agruparon las preguntas de investigación, una vez que llevé a cabo el análisis e interpretación de las mismas, la estructuré bajo los siguientes títulos:

- La docente del primer año: características personales y calidades académicas.

- La docente y su desarrollo profesional: momentos y espacios aprovechados para su enriquecimiento.

- La planificación y ejecución de las actividades de lectura y de escritura: la hebra que hilvanó las situaciones de aprendizaje del primer año.

- La escuela por dentro: una aproximación necesaria en el desarrollo de la presente investigación.

- Las características de los niños y las niñas y los procesos de aprendizaje.

Es importante destacar que cada título agrupa subtítulos bajo los cuales describo, analizo e interpreto eventos relacionados con el proceso de enseñanza y de aprendizaje de la lengua escrita tal y como fueron desarrollados en el aula, siguiendo la línea del tiempo dada por los meses del curso lectivo y un proceso histórico como lo es el orientar a los alumnos y a las alumnas en la adquisición de la lengua escrita. Por consiguiente, presento situaciones de aprendizaje desarrolladas en el aula donde incorporo metáforas, creencias, proceso metodológico así como las voces de la docente y de los niños y de las niñas para responder a las características del diseño que orientó la presente investigación; un diseño de carácter cualitativo y etnográfico.

Tal y como puede observarse el carácter flexible del diseño de la investigación y de las herramientas utilizadas en el proceso, me permitieron ordenar los datos y de esa manera, generar estructuras que me hicieran posible, como investigadora, descubrir lo que subyace en ellos. Es en esa búsqueda, donde el investigador crea formas de organización acordes con los datos que cuenta y con la visión que posee de las cosas que investiga así como de la relación de éstas con el entorno.

Seguidamente presento el esquema que me orientó para organizar los datos racabados en la Matriz No 2

Organización de la información en la matriz

\begin{tabular}{lll}
\hline Aprestamiento & $\begin{array}{l}\text { Actividades } \\
\text { iniciales } \\
\text { de escritura }\end{array}$ & Trabajo con textos \\
Febrero & Marzo & Octubre Diciembre \\
\hline
\end{tabular}

Otro aspecto por destacar en el análisis de la información es el hecho de que las observaciones fueron analizadas con la directora de la investigación. Ese espacio constituyó un momento sumamente valioso ya que logré una realimentación constante del proceso. Del comentario realizado surgieron nuevas ideas, también la necesidad de fijar la atención en determinados aspectos del trabajo, ir tras la búsqueda de nueva bibliografía con la cual poder apoyar lo observado. Asimismo me permitió el poder ubicar lo anotado y analizado en la perspectiva de la investigación.

Como parte del proceso metodológico que fui construyendo en el presente estudio, es necesario destacar la integración de las investigadoras, estudiantes del Programa de Doctorado, que realizamos estudios en el aula. Ese equipo lo integramos tres estudiantes y la directora de nuestras investigaciones. La conformación de ese equipo de trabajo nos permitió un enriquecimiento constante.

\section{La búsqueda de una ruta que me permitiera la construcción de significados: una vivencia fascinante en mi labor como investigadora}

Mi labor como investigadora, deseosa de develar desde el aula los aspectos que facilitan o dificultan la innovación de los procesos de lectura y escritura en un primer año 
del I Ciclo, fue fascinante. Lo anterior debido a que una vez organizada la información en la matriz $\mathrm{N}^{\circ} 2$ tuve que diseñar la ruta o las rutas para analizar e interpretar la información que poseía. El proceso de organización de la información con base en las categorías me llevó a construir cinco apartados en lugar de seis tal y como los diseñé originalmente.

En esos apartados y de acuerdo con el tipo de investigación que llevé a cabo, describo situaciones de aprendizaje, materiales, el espacio del aula asimismo presento las voces de los protagonistas y de las protagonistas del estudio llevado a cabo y algunas fotografías que ilustren la información ahí presentada. Cada uno de esos apartados constituye una unidad en sí misma pero a la vez, sé interrelaciona con los otros, lo anterior con el propósito de dar unidad a la información recabada durante la investigación.

En la elaboración de cada uno de esos apartados traté de incorporar los siguientes aspectos: una descripción de las situaciones de aprendizaje desarrolladas en el aula, la confrontación de lo observado con la teoría que he encontrado al respecto, comentarios analíticos en relación con esos dos aspectos, reflexiones y nuevas interrogantes surgidas en el proceso de construcción de esos apartados pues como sabemos la investigación no concluye con un informe, por el contrario, genera inquietudes que pueden ser motivo de otros estudios. Asimismo incluyo algunas recomendaciones que permitan dar un cierre a cada uno de ellos, información que sirvió de base para construir los hallazgos, conclusiones y recomendaciones del presente estudio que quiero compartir con los lectores al finalizar el presente artículo:

La necesidad de incorporar una nueva área de trabajo durante el desarrollo de la investigación. Esta emergió de las observaciones realizadas y se relaciona con el trabajo en el aula e ir seleccionando las preguntas orientadoras del estudio que fueron planteadas al inicio del mismo e incorporar las preguntas que emergían en el proceso.

Asimismo la evolución del proceso de investigación me llevó de una investigación etnográfica, interpretativa tal y como la había diseñado en un principio a una investiga- ción acción. Lo anterior debido a inquietudes, necesidades que la docente me planteaba y que le permitían reorientar su labor hacia un trabajo más creativo, más acorde con los estudiantes y las estudiantes, con el contexto y por lo tanto, considerar las innovaciones en cuanto a los procesos de lectura y escritura en el Primer año del I Ciclo. Valorar los aportes de la investigación hermeneútica y fenomenológica en la investigación llevada a cabo. Esos dos tipos de investigación me brindaron elementos para analizar los datos que emergieron durante ese proceso rescatando el carácter histórico y contextual de ellos.

Aprovechar las oportunidades de aprender que brinda la escuela para diseñar propuestas de capacitación y actualización de docentes que les sean significativas pues el espacio del aula, es un lugar único, donde se construye una cultura y se lleva a cabo un intercambio de significados. Comprender que los procesos de lectura y escritura son procesos sociohistóricos pues tienen lugar en un espacio y un momento determinados para así generar situaciones de aprendizaje acordes con el entorno cultural en que se encuentran los aprendices, es decir, tomar en cuenta su "capital cultural".

Comparar el desarrollo que siguen los métodos para la atención de los procesos de lectura y escritura, con el desenvolvimiento de los hilos de una madeja, que se van desarrollando poco a poco, bajo la orientación del docente o de la docente. Esos hilos a su vez, se van entrelazando con otros, propios del quehacer pedagógico de ese nivel del sistema educativo.

La labor del aula se orienta mediante un horario mosaico, por asignaturas lo que impide dar secuencia a las situaciones de aprendizaje. Asimismo se dio una descontextualización de las situaciones de aprendizaje lo que impide que éstas sean significativas así como tomar los conocimientos previos como punto de partida para los nuevos aprendizajes.

Captar la preferencia de los niños y de las niñas por las asignatura de español y las asignaturas especiales del curriculum no así por las otras asignaturas instrumentales.

Falta el acompañamiento a la maestra por parte de las autoridades del Ministerio de Educación Pública lo que le lleva a trabajar en forma aislada, en solitario sin una realimentación teórico práctica del proceso que se lleva a cabo.

Una diversidad en el aula que es necesario comprender para poder atender a los niños y niñas de acuerdo con la variedad que respecto a aspectos como el cultural, social, económico, estilos de aprendizaje presentan los estudiantes y las estudiantes. Aproximación básica en los procesos de globalización que sufre nuestra sociedad. Un clima cálido tanto en el aula como en la escuela que facilitó el desarrollo de la investigación.

El deseo de la docente por aprender, aspecto que se debe potenciar para su desarrollo y el de los niños y niñas con quienes interactúa en los procesos del aula. La preocupación de la maestra por la formación integral de los niños y de las niñas, situación que demuestra en cada momento del trabajo. 
La necesidad de una coordinación de los grupos por nivel a la que no se le dio secuencia en el tiempo pues las docentes de Primer año utilizaban diferentes métodos para la enseñanza y aprendizaje de la lectura y escritura. Esa coordinación habría sido muy provechosa pues el intercambio favorece la construcción social del conocimiento y el fortalecimiento de los procesos innovadores.

Es mi deseo que la lectura del presente artículo permita la reflexión acerca de las posibilidades de aprender que ofrece la escuela y que nuestras propuestas de mejoramiento de la educación costarricense sean construidas con base en las realidades de cada contexto de aprendizaje.

\section{Agradecimiento}

Deseo expresar mi más sincero agradecimiento a la Dra. Natalia Campos Saborío y al Dr. Gilberto Alfaro Varela por los aportes así como por la orientación que me brindaron durante el desarrollo de la investigación en que se sustenta este artículo.

\section{Referencias bibliográficas}

Abarca, Sonia. Metáforas de los docentes acerca del aprendizaje: del lenguaje simbólico a la práctica concreta. En: ABRA, Revista de la Facultad de Ciencias Sociales. $\mathrm{N}^{\circ}$ 23-24. Heredia: EUNA. pp. 75-81. 1997.

Alfaro, Gilberto, Gallard, Alejandro, Tobin, Kenneth. Creencias, metáforas y metonimias que limitan las posibilidades de cambio a los maestros. Ponencia presentada en el Seminario sobre Investigación Cualitativa en la Educación Latinoamericana. San José, Costa Rica. 15p. 1991.

Bettelheim, Bruno y Zelan, Karen. Aprender a leer (fragmento). En: lectura y Vida. Revista Latinoamericana de Lectura. Año 8. No 4. Buenos Aires: IRA. pp. 29-31. 1986.
Carr, Wilfred. Una teoría para la educación. Hacia una investigación crítica. Madrid: Morata y La Coruña: Fundación Paidea. 173 p. 1996.

Colas Bravo, María del Pilar y Buendía Eisman, Leonor. Investigación educativa. Sevilla: ALFAR. pp. 43-107. 1994.

Del Rincón, Delio y colaboradores. Técnicas de Investigación en las Ciencias Sociales. 426 p. 1995.

Dobles, María Cecilia y colaboradoras. Investigación en educación. Procesos. Interacciones. Construcciones. San José: EUNED. 223 p. 1996.

Edwards, Verónica. Los sujetos y la construcción social del conocimiento escolar en primaria: Un estudio etnográfico. En: Aportes. Bogotá: Dimensión Educativa. pp. 19-22. 190p. 1991.

Elliot, J. El cambio educativo desde la investigación acción. Madrid: Morata. 190 p. 1996.

La investigación acción en educación. Madrid: Morata. 329 p. 1997.

Fernández, Miguel. La profesionalización del docente. Perfeccionamiento. Investigación en el aula. Análisis de la práctica. Madrid: Siglo XXI. 243p. 1995.

García, Nidia y colaboradoras. Conocimiento, participación y cambio. Capacitación de docentes a partir de la investigación en el aula. 1. Ed. Editorial de la Universidad de Costa Rica. San José. 275 p. 1993.

Conocimiento, participación y cambio. Espacio en el aula. 1. Ed. Editorial de la Universidad de Costa Rica. San José. 140 p. 1993. 
Conocimiento, participación y cambio. Comportamientos en el aula. 1. Edición. Editorial de la Universidad de Costa Rica. San José. 305 p. 1993.

Gibaja, Regina Elena. La cultura de la escuela. Buenos Aires: Aique. 115 p. 1992.

Gimeno, J. Comprender y transformar la enseñanza. Madrid: Morata. 445 p. 1993.

Goetz, J.P. y Le Compte, M.D. Etnografía y diseño cualitativo en investigación educativa. Madrid: Morata. 279 p. 1988.

Henao, Octavio. Influencia de esquemas culturales específicos en la comprensión del lenguaje metafórico. En: Lectura y Vida. Revista Latinoamericana de Lectura. Año 7. $\mathrm{N}^{\circ}$ 1. Buenos Aires: IRA. pp. 25-29. 1986.

Martínez, Miguel. Comportamiento bumano: nuevos métodos de investigación. México: Trillas. 278p. 1989.

Maxwell, Joseph A. Qualitative Research Design an Interactive Approach. California, U.S.A.: Sage Publications. 153 p. 1996.

Olson, Mary (comp.). La investigación acción entra al aula. Buenos Aires: Aique. 164 p. 1991.

Rey, Roberto y Santamaría, Juana. El proyecto educativo de centro. Madrid: Escuela Español S.A. 324 p. 1992.

Rockwell, Elsie. Etnografía y teoría de la investigación educativa. En: Aportes. Germán Mariño. Comp. Bogotá: Dimensión Educativa. pp. 5-18. 1991.

Rueda Beltrán, Mario y Campos, Miguel Ángel. Investigación etnográfica en educación. México D.F.: UNAM. 397 p. 1992.
Rueda Beltrán, Mario y colaboradores. La etnografía en educación. Panorama, prácticas y problemas. México D.F.: CISE-UNAM. 623 p. 1994.

Shagoury Hubbard, Ruth y Miller Power, Brenda. The Art of Classroom Inquiry. A hanbook for teachers-researchers. New Hampshire. U.S.A.: Heinemann. 171p. 1993.

Sánchez, Marta Eugenia. Una nueva mirada a los procesos de lectura y escritura. Tesis para optar por el Grado de Doctora en Educación. Universidad Estatal a Distancia. Sistema de Estudios de Posgrado. San José. 284p. 2000.

Sierra Bravo, R. Técnicas generales del trabajo de investigación científica. En: Tesis doctorales y trabajo de investigación científica. Madrid: Paraninfo. S.A. pp. 53-72. 1996.

Técnicas del trabajo intelectual. En: Tesis doctorales y trabajo de investigación científica. Madrid: Paraninfo S.A. pp. 73-106. 1996.

La creatividad. En: Tesis doctorales y trabajo de investigación científica. Madrid: Paraninfo. S.A. pp. 107-125. 1996.

Stake, R.E. Investigación con estudio de casos. Madrid: Morata. 159 p. 1998.

Taylor, S.J. y Bodgan, R. Introducción a los métodos cualitativos de investigación. Barcelona: Paidós, S.A. 343 p. 1996.

Valles, Miguel. Técnicas cualitativas de investigación social. Reflexión metodológica y práctica profesional. Madrid: Síntesis S.A. pp. 47-105. 1997.

Van dalen, D.B. y Meyer, W.J. Manual de Técnica de la Investigación Educacional. Buenos Aires: Paidós. 542 p. 1979. 
Wittrock, Merlin. La investigación de la enseñan$z a$, II. Métodos cualitativos y de observación. Madrid: Paidós-M.E.C. 431 p. 1989.

La investigación de la enseñan$z a$, III. Profesores y alumnos. Madrid: Paidós - M.E.C. pp. 437-721. 1997.

Woods, Peter. La escuela por dentro. La etnografía en la investigación educativa. Madrid: Paidós-M.E.C. 220 p. 1989.

\section{Otros materiales}

Campos, Natalia. El paradigma cualitativo en la investigación educativa. Vídeo conferencia $\mathrm{N}^{\circ}$ 2. San José: Universidad Estatal a Distancia. Sistema de Estudios de Posgrado. 1998.
El método etnográfico en la investigación cualitativa. Vídeo Conferencia $\mathrm{N}^{\circ}$ 4. San José: Universidad Estatal a Distancia. Sistema de Estudios de Posgrado. 1998.

Villalobos, Luis Ricardo. El investigador educativo ante los caminos de la ciencia. Vídeo Conferencia. San José: Universidad Estatal a Distancia. Sistema de Estudios de Posgrado. 1998.

Investigación cuantitativa en el campo educativo, Paradigma positivista. Vídeo Conferencia. San José: Universidad Estatal a Distancia. Sistema de Estudios de Posgrado. 1998. 